\title{
Erratum to: Inflammation in Patients with Schizophrenia: The Therapeutic Benefits of Risperidone Plus Add-On Dextromethorphan
}

\author{
Shiou-Lan Chen • Sheng-Yu Lee • Yun-Hsuan Chang • \\ Shih-Heng Chen • Chun-Hsieh Chu • \\ Nian-Sheng Tzeng • I-Hui Lee $\cdot$ Po-See Chen • \\ Tzung Lieh Yeh • San-Yuan Huang • Yen-Kuang Yang • \\ Ru-Band Lu • Jau-Shyong Hong
}

Published online: 25 July 2012

(C) Springer Science+Business Media, LLC 2012

Erratum to: J Neuroimmune Pharmacol (2012)

DOI 10.1007/s11481-012-9382-z

The authors would like to correct an error in publication. This was in the omission of Hong JS. Hong JS's affiliation is in the Neuropharmacology Section, Laboratory of Pharmacology, National Institute of Environmental Health Sciences, National Institutes of Health, USA

The online version of the original article can be found at http://dx.doi.org/ 10.1007/s11481-012-9382-z.

S.-L. Chen · Y.-H. Chang • R.-B. Lu

Institute of Behavioral Medicine,

Tainan, Taiwan

S.-L. Chen $\cdot$ S.-Y. Lee $\cdot$ S.-H. Chen $\cdot$ C.-H. Chu $\cdot$ I.-H. Lee $\cdot$

P.-S. Chen $\cdot$ T. L. Yeh $\cdot$ Y.-K. Yang $\cdot$ R.-B. Lu

Department of Psychiatry,

National Cheng Kung University Hospital,

Tainan, Taiwan

\section{Y.-H. Chang • R.-B. Lu}

Institute of Allied Health Sciences, College of Medicine,

National Cheng Kung University,

Tainan, Taiwan

S.-L. Chen $\cdot$ S.-Y. Lee $\cdot$ S.-H. Chen $\cdot$ C.-H. Chu $\cdot$ I.-H. Lee

P.-S. Chen · T. L. Yeh • Y.-K. Yang • R.-B. Lu

Addiction Research Center, National Cheng Kung University,

Tainan, Taiwan
N.-S. Tzeng $\cdot$ S.-Y. Huang

Department of Psychiatry, Tri-Service General Hospital,

National Defense Medical Center,

Taipei, Taiwan

R.-B. Lu $(\bowtie)$

Department of Psychiatry, College of Medicine and Hospital,

National Cheng Kung University,

138 Sheng-Li Road,

Tainan 70428, Taiwan

e-mail: rblu@mail.ncku.edu.tw

R.-B. Lu

e-mail: rubandlu@gmail.com

\section{J.-S. Hong}

Neuropharmacology Section, Laboratory of Pharmacology,

National Institute of Environmental Health Sciences,

National Institutes of Health,

Research Triangle Park, NC 27709, USA 